\title{
The Correlation between Gene Polymorphism and Hepatocellular Carcinoma
}

\author{
Zhao-Chun Chi, Chang-Xin Geng and Quan-Jiang Dong
}

The association of gene polymorphism and susceptibility to hepatocellular carcinoma (HCC) has been widely studied in recent years. Gene mutations are closely related to HCC. Understanding and measuring the gene mutations are useful to reduce the incidence of HCC and improve its prognosis.

Key words: Susceptibility; Genes; Hepatocellular carcinoma; Gene polymorphism

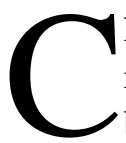
hronic hepatitis B virus (HBV) infection is related to hepatocellular carcinoma (HCC), but the mechanism remains unclear. A great deal of researches has been conducted to explore the relationship between gene polymorphism and HCC in recent years. Many studies indicated that gene mutations and HCC are closely related. These researches mostly are carried out in Asia, and the subjects, size and results are prominent especially in China. They put forward a new idea for diagnosis and treatment of HCC, and provide a new direction for further research on how to reduce the risk of liver cancer, and how to treat chronic hepatitis B and liver cancer. If HCC susceptible populations were screened in time, and were given intervention and preventive measures early, the incidence of HCC would be expected to reduce.

\section{STAT4 AND LEUKOCYTE ANTIGEN GENE MUTATION AND HCC}

STAT4 (signal transducer and activator of transcription factor 4) is a signal transducer and activator of transcription factor, and HLA-DQ is a leukocyte antigen DQ gene. Now, it's obviously that they are involved in the pathogenesis of HCC. In a Chinese study, 11799 blood cell DNA samples of independent people with chronic hepatitis B in 7 areas in China were collected by Genetics Institute of Fudan University, including 5480 patients with HCC, 6319 controls with a history of hepatitis B but

\footnotetext{
Department of Gastroenterology, Qingdao Municipal Hospital, Affiliated Hospital of Ocean University of China, Qingdao, Shandong Province 266071, China

Correspondence: Zhao-chun Chi, E-mail: c.z.chow@163.com
}

without HCC. Using genome-wide association study (GWAS) techniques, the alleles frequency of nearly 730000 single nucleotide polymorphism (SNP) in the entire gene sequence of two groups were compared and analyzed. Eventually hepatitis B - related cancer susceptibility gene loci on the STAT4 gene and HLA-DQ gene cluster were identified. The result showed STAT4 and HLA-DQ gene were the key susceptibility genes of hepatitis B patients suffering from liver cancer. Jiang et al studied 2514 cases of chronic HBV carriers, including 1161 cases of HCC, 1353 cases of controls, and the other 6 independent populations, including 4319 patients with HCC, 4966 cases of controls. Conjoint analysis indicated that STAT4 rs7574865 loci and HLA-DQR rs9275319 loci had a significant correlation with $\mathrm{HCC}$, the former $P$ was $2.48 \times 10^{-10}, O R=1.21$, the latter $P$ was $2.72 \times 10^{-17}$, $O R=1.49{ }^{1}$

Human leukocyte antigen DP-DQ gene mutation has effects on the clearance of HBV and occurrence of HCC. A recent GWAS research found that HLADP (rs3077 and rs9277535) and HLA-DQ (rs2856718 and rs7453920) were associated with HCC in patients with chronic HBV infection. Hu et al studied 1300 cases of HCC patients with positive HBV, 1344 cases of HBV carriers, 1344 cases of patients with spontaneous HBV clearance, and found that HLADP rs9277535, HLA-DQ rs7453920 and rs2856718 could affect the clearance of HBV. ${ }^{2}$ Besides, HLA-DP rs3077 showed significant susceptibility to persistent infection of HBV and occurrence of HCC.

The correlation between HLA II gene and HCC has certain differences in different nations and races. 
There was a significant correlation between HLA II DRB1*04, DQB1*02 and HCC in Egypt. HLA II DRB $1 * 04$ and DQB1*02 were risk factors of HCC, while DQB1*06 might be a protective gene. ${ }^{3}$ Jin et al found that the frequency of HLA-DRB1*140101 allele in HCC patients was significant higher than that of non-cancer group. ${ }^{4}$ Patients with HLA-DRB1*140101 allele had a higher cumulative incidence of HCC than patients without it in the 2th, 4th, 6th year. They drew a conclusion that HLA-DRB1*140101 was a potential risk factor for the patients with chronic HBV infection to deteriorate into HCC.

\section{P53 AND MDM2 POLYMORPHISMS AND HCC}

The p53 (tumor suppressor gene) signal pathway is a powerful barrier for the progression of tumors. p53 has 2 SNPs loci, p53 codon 72Arg (arginine) 72Pro (proline) and MDM2 (murine double minute 2) SNP309 ( $\mathrm{T}>\mathrm{G}$, deoxythymidine $>$ deoxyguanosine), which can induce dysfunction of p53. But the risk of two SNPs in HCC is not consistent. MDM2, one of the oncogene, is a ubiquitin protein ligase, which is composed of 698 adenine, 491 cytosine, 541 guanine, 624 thymine. It consists of five different types, MDM2 $\mathrm{a}, \mathrm{b}, \mathrm{c}, \mathrm{d}$ and e. It is not only one of the target gene transcriptionally regulated by p53, but also an important regulative factor of p53. Yang et al studied 350 cases of HCC, 230 cases of patients without HCC, 96 cases of healthy controls. The results showed that p53 Pro/Pro and MDM $2 \mathrm{G} / \mathrm{G}$ had a significant correlation with $\mathrm{HCC}$ $(P=0.047)$, and they were the independent factors influencing the recurrence and survival of HCC $(P<$ $0.05) .{ }^{5}$ Yang et al also found that the patients with p53 Pro/Pro and MDM2 G/G genotype simultaneously had poorer prognosis than those with other genotype. The DFS (disease-free survival) and OS (overall survival) were significantly different. Patients with p53 Pro/Pro and MDM2 G/G genotype simultaneously had poorer prognosis compared with those with other genotypes in A stage $(P<0.05)$. Thus, the combined Pro/Pro and MDM2 G/G genotype is associated with increased risk of developing HCC and is an independent adverse prognostic indicator in early stage HCC. A case-control study in Turkey suggested that the p53 Pro/Pro homozygotes, especially in male patients with HBV infection was the susceptible gene to HCC. ${ }^{6}$ South Korean scholars pointed out that MDM2 SNP 309 and p53Arg72Pro were associated with early
HCC in patients with chronic HBV infection.

SNP $309 \mathrm{~T}>\mathrm{G}$ at MDM2 promoter can affect expression of MDM2 protein and tumorigenesis. Logistic regression analysis showed that HBV-related HCC patients had significant different deletion of MDM2 SNP 309 compared with healthy controls or non-HCC patients with HBV infection, and also revealed that patients with $G$ allele had a higher incidence of HCC than patients with T allele. ${ }^{8,9}$

\section{THE MIRNA POLYMORPHISM AND HCC}

MiRNAs (micro RNAs) 371-373 is originated from the transcription of former miRNA. There is upregulation of miRNA in HBV-related HCC patients, and miRNA can regulate HBV infection. Kwak et al used TaqMan probe to study the relationship of primary miRNAs (pri-miRNAs) 371-373 polymorphism with occurrence of HCC and clearance of HBV. The results showed that among chronic carriers and liver cirrhosis patients, the A allele of rs3859501 and the haplotype primiRNAs-371-373_ht2 were more protective to HCC than other genotypes and haplotypes. ${ }^{10}$

One study found that pri-miRNA-196a functional polymorphism could lead to susceptibility to HCC. MiRNAs are non-protein coding RNAs. Abnormal expression and structural changes of miRNA are involved in the occurrence and development of cancer. The SNP in pri-miRNA can change the miRNA's processing, expression and/or binding to target miRNA, then cause tumor. Recent research indicated that miRNA-196 a2 rs11614913 $(\mathrm{C} \rightarrow \mathrm{T})$ could change the expression of miRNA-196a2 and bind to the target miRNA. The results showed that miRNA-196-a2 rs11614913 could increase the risk of HCC (especially in female patients with HBV infection). It was identified as a susceptibility gene of HCC. ${ }^{11,12}$

\section{DNA DOUBLE STRAND BREAK REPAIR GENE XRCC5 POLYMORPHISMS AND HCC}

XRCC (X-ray repair cross complementing) genes contain seven kinds. As a member of RAD51 family, it is the repair gene of DNA double strand breaks. DNA damages caused by environmental risk factors are not repaired accurately, which induces chromosomal aberration, genomic instability and occurrence of HCC. XRCC5 gene mutation may 
lead to susceptibility to HCC. Studies had found that XRCC5 rs16855458 could significantly reduce the risk of HCC, while XRCC5 rs9288516 could significantly increase the risk of HCC, and the role of them was more significant in patients with HBV infection than patients without HBV infection. Haplotype analysis showed that XRCC5 gene had one risk haplotype "AAATC" and two protective haplotypes "GGGTT", and the latter reduced the risk of HCC. ${ }^{13}$ So mutation of XRCC5 gene may play a role in susceptibility to HCC.

A research found that XRCC1 gene C.1161 G > A and C.1799 C > G were associated with increased risk of HCC in Chinese population using PCR-RFLP. As for the C.1161G > A, the AA genotype was statistically associated with the increased risk of HCC compared to GG wild genotype. As for the C.1779C > G, the risk of HCC was significantly higher for GG genotype compared to CC wild genotype. ${ }^{14} \mathrm{~A}$ study on the relationship of the XRCC4 coding region polymorphisms with Aspergillus flavus B1 associated HCC indicated that XRCC4 rs28383151 AA genotype increased the risk of HCC. It may be a potential risk gene, and can be used as a biochemical marker of HCC prognosis. ${ }^{15}$

\section{CORTICOTROPIN-RELEASING HORMONE RECEPTOR 2 GENE INTRON POLYMORPHISM AND HCC}

Corticotropin-releasing hormone receptor 2 (CRHR2) works in the central and peripheral nervous system. Urocortin (Ucns) and corticotropin releasing hormone (CRH), as the ligand of CRHR2, can mediate inflammatory reaction and inhibit angiogenesis. The recent study found CRHR2 expresseed in many human cancer cells, and was found in the patients with chronic hepatitis $\mathrm{C}$ virus infection. CRHR2 gene rs2267716 polymorphism is related to HCC susceptibility. Using PCR-LDR (ligation detection reaction), Gu et al studied 364 cases of HBV-related HCC, 196 cases of non-HCC patients with HBV infection and 404 cases of healthy controls, and found that significant differences of rs2267716 allele were detected between HBV-related HCC patients and healthy controls or non-HCC patients with HBV infection. ${ }^{16}$ CRHR2 gene rs2267716 polymorphism may increase the risk of developing HCC.

MDR1 GENE POLYMORPHISM AND HCC MDR1 (multidrug resistance 1) gene is an important candidate gene that affects the susceptibility to HCC. Gao et al studied 353 cases of HCC and 335 cases of controls, and the results indicated that MDR1 gene polymorphism was associated with the risk of $\mathrm{HCC}$, but this needs to be confirmed by a large casecontrol study. ${ }^{17}$ Using CRS-PCR (created restriction site-polymerase chain reaction), Yang et al found that the C.1465 C > T polymorphism increased the risk of HCC, and drew a conclusion that MDR1 gene mutation was a valuable molecular marker of HCC. ${ }^{18}$

\section{CYP2E1, HOGG1 AND XRCC1 AND LIVER DISEASE AND HCC}

CYP2E1 (cytochrome P450 2E1) can increase the risk of HBV related liver diseases by 2.68 times, increased HCC by 3.981 times, and is associated with higher histology activity index (HAI) in chronic HBV infected patients. The mutation of CYP2E1 and HOGG1 [8-oxidation of guanine DNA glycosylase (DNA damage repair gene)] can promote liver fibrosis. CYP2E1 combined with XRCC1 gene or HOGG1 combined with XRCC1 gene, both can increase the risk of HCC. If the above three kinds of genes have combination of mutation, the interaction of three genes may make the susceptibility to HCC significantly increase. ${ }^{19}$

\section{OTHER GENE POLYMORPHISM AND HCC}

Genes which can promote or inhibit the occurrence and development of HCC also include Tolllike receptors polymorphism, $\mathrm{mEH}$ (microsomal epoxide hydrolase) polymorphism, GST (glutathione S-transferase) polymorphism, EGF (epidermal growth factor) polymorphism, KIF1B (kinesin like protein) polymorphism, RASSF1A (ras-association domain family 1A) high methylation, RAD51 (homologous recombination repair gene), TGFBR3 (transforming growth factor type III receptor) polymorphism, GRP78 (glucose regulated protein 78) polymorphism, leukocyte telomere length, aromatase (CYP19) promoter polymorphism, methylenetetrahydrofolate reductase (MTHFR) and methionine synthase reductase (MTRR) polymorphism, UDP glucuronosyltransferase (UGT) 1A7 haplotype and so on.

\section{CONCLUSIONS}

Numerous gene polymorphisms are associated with 
the occurrence, development and prognosis of HCC, but most of these gene mutations are not unique to HCC, and they also can occur in many tumors of other parts of the body, so their specificity is poor. Most of the current studies have small number of cases. It requires large scale, multi-country, multi-ethnic and multi-region studies to further determine the clinical value of these gene polymorphisms. At present, the role of these gene polymorphisms in the diagnosis and treatment of HCC needs further study. As time goes on, more gene polymorphisms associated with HCC will be identified. We expect to have a new breakthrough in the prevention and treatment of HCC with the more understanding to the pathogenesis of HCC.

\section{REFERENCES}

1. Jiang DK, Sun J, Cao G, Liu Y, Lin D, Gao YZ, et al. Genetic variants in STAT4 and HLA-DQ genes confer risk of hepatitis B virus-related hepatocellular carcinoma. Nat Genet 2013;45:72-75

2. Hu L, Zhai X, Liu J, Chu M, Pan S, Jiang J, et al. Genetic variants in human leukocyte antigen/DP-DQ influence both hepatitis B virus clearance and hepatocellular carcinoma development. Hepatology 2012;55:1426-1431.

3. El-Chennawi FA, Auf FA, Metwally SS, Mosaad YM, El-Wahab MA, Tawhid ZE. HLA-class II alleles in Egyptian patients with hepatocellular carcinoma. Immunol Invest 2008;37:661-674.

4. Jin YJ, Shim JH, Chung YH, Kim JA, Choi JG, Park WH, et al. Relationship of HLA-DRB1 alleles with hepatocellular carcinoma development in chronic hepatitis B patients. J Clin Gastroenterol 2012;46:420-426.

5. Yang Y, Xia T, Li N, Zhang J, Yang Y, Cong W, et al. Combined effects of p53 and MDM2 polymorphisms on susceptibility and surgical prognosis in hepatitis B virus-related hepatocellular carcinoma. Protein Cell 2013;4:71-81.

6. Sumbul AT, Akkiz H, Bayram S, Bekar A, Akgollu E, Sandikci M. p53 codon 72 polymorphism is associated with susceptibility to hepatocellular carcinoma in the Turkish population: a casecontrol study. Mol Biol Rep 2012;39:1639-1647.

7. Yoon YJ, Chang HY, Ahn SH, Kim JK, Park YK, Kang DR, et al. MDM2 and p53 polymorphisms are associated with the development of hepatocellular carcinoma in patients with chronic hepatitis B virus infection. Carcinogenesis
2008;29:1192-1196.

8. Wang X, Zhang X, Qiu B, Tang Y, Sun H, Ji H, et al. MDM2 SNP309 $\mathrm{T}>\mathrm{G}$ polymorphism increases susceptibility to hepatitis $B$ virus-related hepatocellular carcinoma in a northeast Han Chinese population. Liver Int 2012;32(7):1172-1178.

9. Ding C, Yu H, Yu H, Qin H. TP53 codon 72 polymorphism with hepatocellular carcinoma: a meta analysis. J Int Med Res 2012;40:446-454.

10. Kwak MS, Lee DH, Cho Y, Cho EJ, Lee JH, Yu SJ, et al. Association of polymorphism in pri-microRNAs-371-372-373 with the occurrence of hepatocellular carcinoma in hepatitis B virus infected patients. PLoS One 2012;7:e41983.

11. Akkız H, Bayram S, Bekar A, Akgöllü E, Ulger Y. A functional polymorphism in pre-microRNA-196a-2 contributes to the susceptibility of hepatocellular carcinoma in a Turkish population: a case-control study. J Viral Hepat.2011;18(7):399-407.

12. Kim WH, Min KT, Jeon YJ, Kwon CI, Ko KH, Park PW, et al. Association study of microRNA polymorphisms with hepatocellular carcinoma in Korean population. Gene 2012;504:92-97.

13. Yue AM, Xie ZB, Guo SP, Wei QD, Yang XW. Implication of polymorphisms in DNA repair genes in prognosis of hepatocellular carcinoma. Asian Pac J Cancer Prev 2013;14:355-358.

14. Deng X, Liang J, Jiang M, Zhou X, Liu H. Association between the C.1161G $>$ A and C.1779C $>\mathrm{G}$ genetic variants of XRCC1 gene and hepatocellular carcinoma risk in Chinese population. Int J Biol Sci 2013;9:289-294.

15. Long XD, Yao JG, Zeng Z, Ma Y, Huang XY, Wei ZH, et al. Polymorphisms in the coding region of $X$-ray repair complementing group 4 and aflatoxin B1-related hepatocellular carcinoma. Hepatology 2013;58:171-181.

16. Gu X, Qi P, Zhou F, Ji Q, Wang H, Dou T, et al. An intronic polymorphism in the corticotropin-releasing hormone receptor 2 gene increases susceptibility to HBV-related hepatocellular carcinoma in Chinese population. Hum Genet 2010;127:75-81.

17. Yang D, Zhou F, Wang X, Gao H, Li G, Xue M. Association analysis between MDR1 gene polymorphisms and risk of hepatocellular carcinoma in Chinese population. Biomarkers 2013;18:236-241.

18. Gao J. Association of MDR1 gene polymorphisms with the risk of hepatocellular carcinoma in the Chinese Han population. Braz J Med Biol Res 2013;46:311-317.

19. Bose S, Tripathi DM, Sukriti, Sakhuja P, Kazim SN, Sarin SK. Genetic polymorphisms of CYP2E1 and DNA repair genes HOGG1 and XRCC1: association with hepatitis B related advanced liver disease and cancer. Gene 2013; 519: 231-237. 